\title{
Sistema de evaluación para la formación a distancia de profesionales
}

\section{del Teso, $\mathbf{R}^{\mathrm{a} 1}$, Estruch, E. ${ }^{\mathrm{a} 2}$, Gómez, E. ${ }^{\mathrm{a} \text {, }}$ Soriano, J. ${ }^{\mathrm{a} 4}$}

${ }^{a}$ ITA, Departamento de Ingeniería Hidráulica y Medio Ambiente. Escuela Técnica Superior de Ingenieros industriales. Universitat Politècnica de València. ${ }^{a 1}$ rodete@ita.upv.es, a2maesjua1@ita.upv.es, ${ }^{\text {a3}}$ elgosel@ita.upv.es, ${ }^{24}$ jasool@ita.upv.es

\begin{abstract}
The online training of students in the field of hydraulic engineering entails the need to undertake periodic self-evaluations to ensure their understanding of the topics developed. The lack of some preliminary key concepts may lead to a deficient understanding in future more complicated developments. Therefore, a solid foundation is crucial to ensure that students understand the most complex concepts of each topic.

To achieve this goal, a self-assessment system has been designed for all elearning courses taught by the ITA, in both posgraduated and specific programs. This system ensures that students reach the specific objectives of each course. This evaluation system has been used since 2010, in the 20 courses taught by the ITA concernint efficient urban water management. More than 1,500 students have been assessed with this system, with a success rate higher than $90 \%$.
\end{abstract}

Keywords: assessment, urban water systems, hydraulics, online learning, online courses, checkpoint, test

\section{Resumen}

La formación a distancia en el campo de la ingeniería conlleva la necesidad de definir un sistema de autoevlación, que permita al alumno asegurarse que va alcanzando los objetivos y la comprensión de los temas desarrollados. No dominar los conceptos clave puede conllevar al no entendimiento de desarrollos posteriores más complejos. Es necesario establecer una base sólida que permita al alumno profundizar poco a poco en aspectos más específicos y elevados.

Para conseguir este objetivo se ha desarrollado para todos los cursos online impartidos por el ITA, tanto de formación superior como de formación específica, un sistema de autoevaluación que permita asegurar que el alumno aquiere los conocimientos específicos de cada asignatura. Este sistema de evaluación se lleva utilizando desde 2010, en los 20 cursos que se imparten desde relacionados con la gestión eficiente del agua urbana, formando a más de 1.500 alumnos con un porcentaje de aprobados superior al $90 \%$.
} 
Palabras clave: evaluación, agua urbana, hidráulica, formación a distancia, cursos online, checkpoint, test.

\section{Introducción}

La metodología usada en formación a distancia es fundamental para asegurar que los alumnos que la superan con éxito han adquirido los conocimientos mínimos requeridos para aprobar (Sun et al., 2006). Más aún cuando esta formación a distancia va enfocada a conseguir un título de posgrado, tal y como ocurre en la formación a distancia impartida por el ITA.

El sistema de evaluación implementado por el ITA para la formación a distancia tiene el objetivo de garantizar que los conocimientos se van adquiriendo y consolidando por parte de los estudiantes a través de una serie de puntos de control, o “checkpoints” que permitirán verificar el grado de compresión del alumno sobre el contenido desarrollado hasta el momento. Al final de cada unidad, además, deberá responder un test en el que se preguntan los conceptos teóricos principales estudiados en la unidad, ya que tener claros estos conceptos le permitirá en un futuro resolver casos prácticos de aplicación. Para reafirmar la comprensión de estos conceptos, una vez finalizados todos los contenidos, el alumno se enfrentará a un test final que reúne preguntas sobre todos los conceptos teóricos y prácticos que se han visto en el curso. Tanto los puntos de control, como los test de las unidades y el test final, forman parte de la evaluación del curso. Además, en los cursos más prácticos, donde el alumno debe resolver problemas, o donde debe trabajar con programas informáticos, se plantea la resolución de una serie de ejercicios prácticos que deberá enviar para que los profesores del curso los evalúen y proporcionen una retroalimentación acera de la idoneidad de la solución propuesta por el alumno.

En este trabajo se presenta el sistema de evaluación utilizado en los módulos de título propio y formación específica impartidos por el ITA. La superación de estos módulos, conlleva la obtención de títulos propios de posgrado como son el Máster en Gestión Eficiente del Agua Urbana, el Diploma de Especialización en Análisis y Diseño de Redes de Agua, el Experto Universitario en EPANET y el Experto Universitario en Transitorios Hidráulicos con Allievi. Títulos de posgrado muy específicos que pueden ofrecer una vía de acceso a puestos laborales especializados, y que por lo tanto, deben asegurar unos conocimientos mínimos para desarrollar labores tan específicas.

Con los cursos de formación a distancia desarrollados por el ITA, de los que el sistema de evaluación que se presenta es una parte fundamental, se ha formado a más de 1.500 alumnos. En los últimos dos años se ha observado una creciente demanda debido a la implantación de los títulos propios denominados anteriormente. La gran mayoría de los alumnos provienen principalmente de Latinoamérica así como de empresas tanto públicas como privadas. Esto hace que la labor de conseguir que el alumno adquiera los conocimientos mínimos exigidos para superar la formación sea imprescindible (Bhuasiri et al., 2011). 


\section{Objetivos}

La evaluación en formaciones a distancia donde existe un elevado número de alumnos suele ser una tarea compleja, ya que el seguimiento de los alumnos por parte de los tutores de la formación se complica conforme el número de alumnos matriculados crece. Por esta razón, es necesario preparar un sistema de evaluación automatizado que permita al alumno conocer su grado de aprendizaje respecto a lo estudiado en el curso. Este aprendizaje no debe evaluarse únicamente al final de la formación, si no durante toda ella, lo que garantiza que el alumno sea consciente de donde está errando, o qué conceptos le resultan más complejos. Esto le permitirá volver a estudiar aquello que no tiene claro o ponerse en contacto con los tutores para pedir apoyo en las tareas que no haya superado.

De esta manera, se trata de conseguir que el alumno adquiera con seguridad los conocimientos básicos desde el comienzo de la formación, para ir profundizando en temas más complejos conforme avanza su estudio.

El objetivo del sistema de evaluación utilizado en la formación a distancia del ITA es conseguir que los alumnos adquieran los conocimientos mínimos requeridos en el curso. Esto se consigue con una evaluación que considera cuatro tipos de tareas distintos: puntos de control o "checkpoints", test de evaluación en cada una de las unidades, test final una vez finalizados los desarrollos del curso y ejercicios prácticos que el alumno debe enviar. Cada una de estas evaluaciones están pensadas de manera diferente para conseguir que una vez el alumno finalice el curso tenga las competencias pertinentes (Frydenberg, 2002). Una vez finalizado el curso, el alumno dispondrá de la capacidad de incorporarse al mercado laboral con unos conocimientos muy específicos sobre hidráulica, modelación de redes o gestión eficiente del agua.

\section{Desarrollo de la innovación}

En la Plataforma de formación Cursosagua, se ha desarrollado cuatro sistemas de evaluación complementarios que permitirán al alumno autoevaluarse a lo largo del estudio del curso. Estos sistemas de evaluación se desarrollan en profundidad a continuación, teniendo pesos diferentes en la nota final del curso en función de las características del mismo.

\subsection{Puntos de control}

Los puntos de control son los más utilizados a lo largo del curso, normalmente aparecerán en dos o más ocasiones en cada unidad, en función de la complejidad y carácter práctico de la formación. En los cursos donde existe mayor información teórica, los puntos de control son menos frecuentes que en aquellos cursos prácticos donde el alumno debe realizar cálculos y resolver problemas planteados paso a paso.

El objetivo de estos puntos de control, es permitir al alumno autoevaluarse a medida que avanza en el estudio del curso, de manera que tras estudiar algún apartado importante el alumno se encontrará ante una evaluación que no podrá saltarse hasta haberla finalizado por completo. Los puntos de control tienen un doble objetivo, por un lado reforzar los conocimientos de los alumnos y que éstos reciban feedback de su trabajo, por otro lado 
mantener activo al alumno durante su estudio (González de la Fuente y Carabantes Alarcón, 2018).

Esta evaluación consistirá en una serie de preguntas teóricas sobre el contenido desarrollado, o prácticas sobre los casos prácticos vistos en la unidad.

En el caso de preguntas teóricas, el alumno seleccionará aquella respuesta que considere correcta de entre una serie de opciones, es decir, se enfrenta a preguntas de selección múltiple con una única respuesta válida. La evaluación de contenidos teóricos se lleva a cabo mediante preguntas multirespuesta debido a las ventajas que ofrece este método de evaluación. Las preguntas multirespuesta son un recurso muy valioso debido a la objetividad de su calificación y la versatilidad que ofrecen para ser aplicables a cualquier cuestión objeto de evaluación (Mendes et al., 2011; Ventouras et al., 2010). Además, este tipo de evaluación permite fácilmente manejar grupos de alumnos numerosos con independencia del lugar en el que se encuentren y el momento en el que realicen el test, cualidades imprescindibles en el aprendizaje a distancia (Singh y (Ruth) de Villiers 2017).

Las opciones de respuesta que los alumnos encontrarán en los puntos de control podrán ser o bien texto o bien imágenes. En el primer caso, la pregunta siempre va acompañada de una imagen que apoya a la pregunta, tal y como se muestra en la Fig. 1.

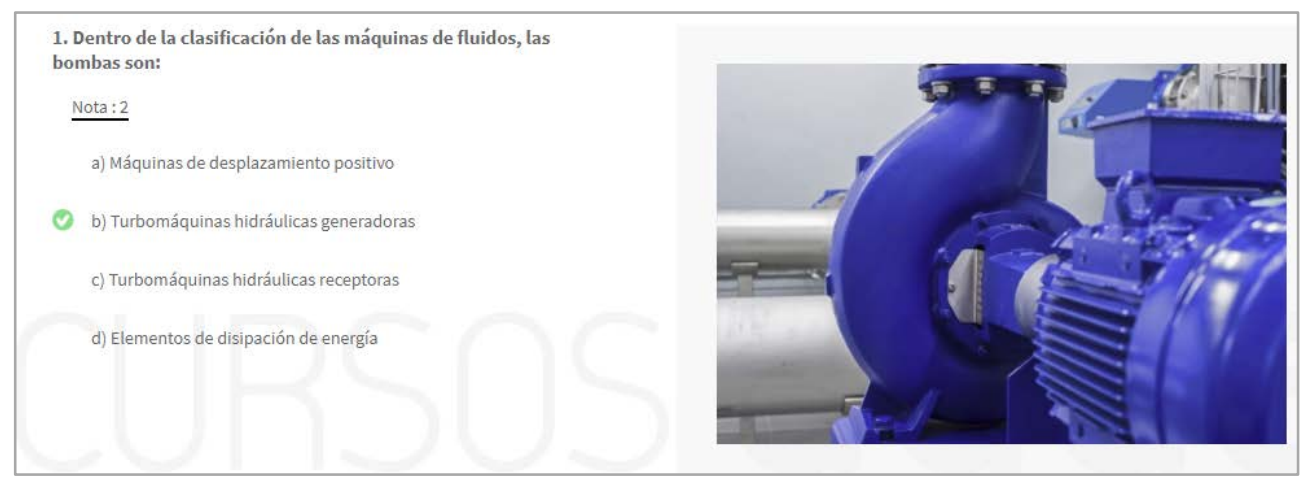

Fig. 1 Pregunta de un punto de chequeo del tipo opción múltiple con texto

En el caso de ser opciones con imágenes, al clicar sobre cada una de las imágenes ésta se amplía de manera que el alumno pueda distinguir sin problemas el contenido de la misma. Este tipo de pregunta suele usarse para reconocer y diferenciar elementos físicos explicados en el curso, como puede ser diferentes tipos de bombas o válvulas. Otro uso frecuente de las preguntas con imágenes es la representación de gráficas. Al alumno se le presentan una serie de gráficos y debe seleccionar aquel que se corresponda con la pregunta planteada, este tipo de preguntas tienen un enfoque más práctico, y suele utilizarse en cursos donde se trabaja con programas informáticos. La Fig. 2 muestra un ejemplo de una pregunta de opción múltiple con imagen. 


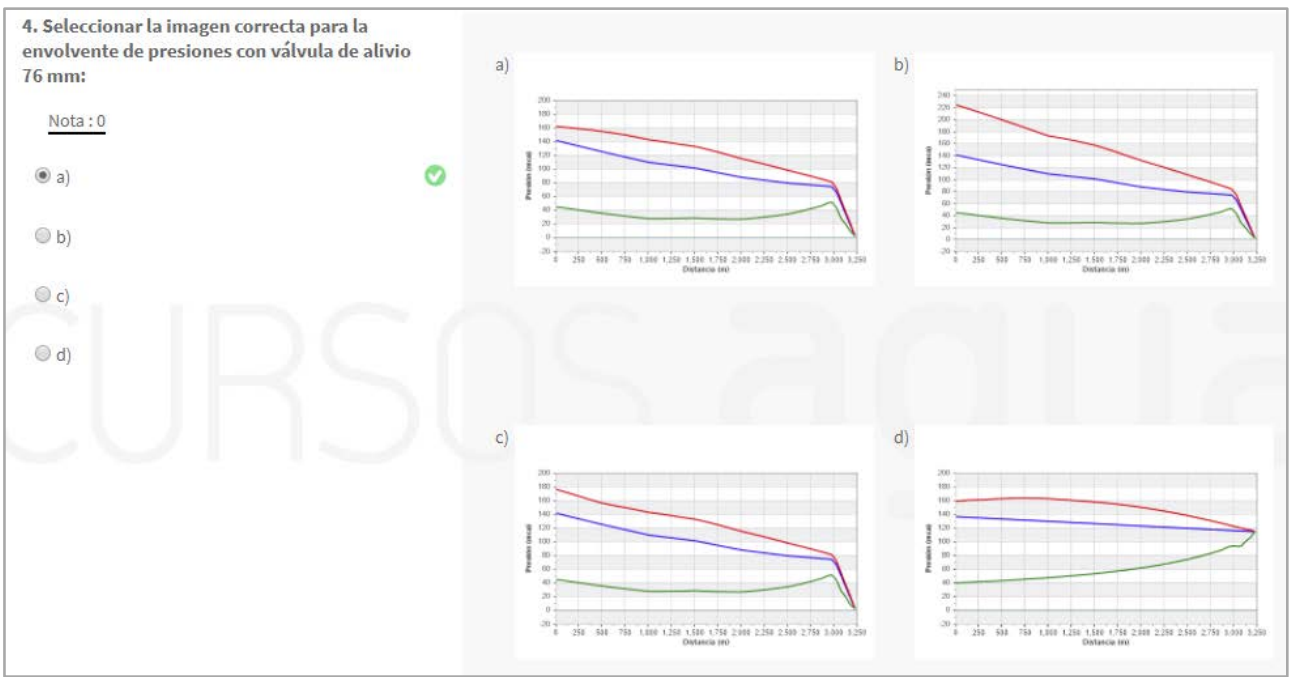

Fig. 2 Pregunta de un punto de chequeo del tipo opción múltiple con imagen

En este tipo de preguntas, el alumno no puede pasar a la siguiente cuestión hasta que no haya seleccionado la respuesta correcta. De manera que cada vez que se contesta de forma errónea, la puntuación de la pregunta baja en función del número de respuestas, hasta ser de cero en el caso de fallar todas las opciones y quedarse únicamente con la válida.

El segundo tipo de pregunta se trata de preguntas numéricas y está enfocada al desarrollo de casos prácticos. Al alumno se le solicita un valor numérico concreto, la pregunta no se dará por correcta hasta que el alumno no introduzca el valor solicitado. Para tener en cuenta los redondeos y posibles pequeños errores en el cálculo, se admite un rango de tolerancia de manera que la pregunta se da por correcta dentro de un rango permitido. Este rango dependerá del orden de magnitud del valor solicitado. En el caso de fallar la respuesta en su primer intento, al alumno se le ofrece una pista sobre la resolución de la cuestión. Esta pista permite orientar al alumno sobre los pasos a seguir y proporciona ayuda acerca de los errores que puede estar cometiendo. De este modo, se proporciona al alumno retroalimentación inmediata que le ayude a encontrar la respuesta correcta (Bailey y Card, 2009). En el siguiente intento, si el valor vuelve a ser erróneo le aparece de nuevo la pista, si vuelve a errar en su tercer intento se le indica el valor correcto de la respuesta para que no se quede bloqueado en la pregunta y pueda continuar con el curso, pero en este caso, su valoración en la pregunta será de cero. Al igual que en el caso de preguntas de selección múltiple, la puntuación de la pregunta disminuye en función del número de intentos fallidos.

En cualquier momento el alumno puede salir del punto de control para buscar la solución de la pregunta, esto obliga al alumno a repasar lo ya estudiado si no sabe la respuesta, asegurando que repasa los contenidos importantes. En caso contrario, la nota de la evaluación será baja y no se superará el curso.

El número de preguntas variará en cada punto de control, dependiendo de la necesidad, pero tratando de no realizar puntos de control muy largos que puedan resultar tediosos al 
alumno. El objetivo es conseguir que el alumno se autoevalúe pero sin llegar a resultarle pesado.

La nota de los puntos de control representa el 30\% de la evaluación final en aquellos cursos donde no existen envíos de ejercicios. Mientras que, en el caso de los cursos con resolución de casos prácticos a evaluar, el peso de los puntos de control es del $10 \%$, ya que se le da un mayor peso a la resolución de ejercicios prácticos, 40\%.

\subsection{Test de unidades}

Al finalizar cada una de las unidades que componen la formación, una vez resueltos los puntos de control y los contenidos teóricos, la plataforma habilita automáticamente un test con diferentes preguntas multirespuesta sobre conceptos teóricos de la unidad. Estas preguntas son del tipo selección múltiple con texto, donde el alumno deberá seleccionar una única respuesta correcta. En este caso, a diferencia de los puntos de control, la pregunta no va acompañada de una imagen que pueda dar indicios de la respuesta y además no se le muestra al alumno si la pregunta contestada ha sido resuelta correctamente, las respuestas correctas y la nota del test se le ofrece una vez finalizado y enviado el mismo. El número de preguntas varía entre unidades en función de la importancia de los conceptos vistos, estableciendo un mínimo de 5 preguntas y un máximo de 10. Cada pregunta errada no descuenta, únicamente se puntúa con cero. Una vez finalizado el test, el alumno puede consultar la nota y los fallos y aciertos realizados tal y como se muestra en la Fig. 3.

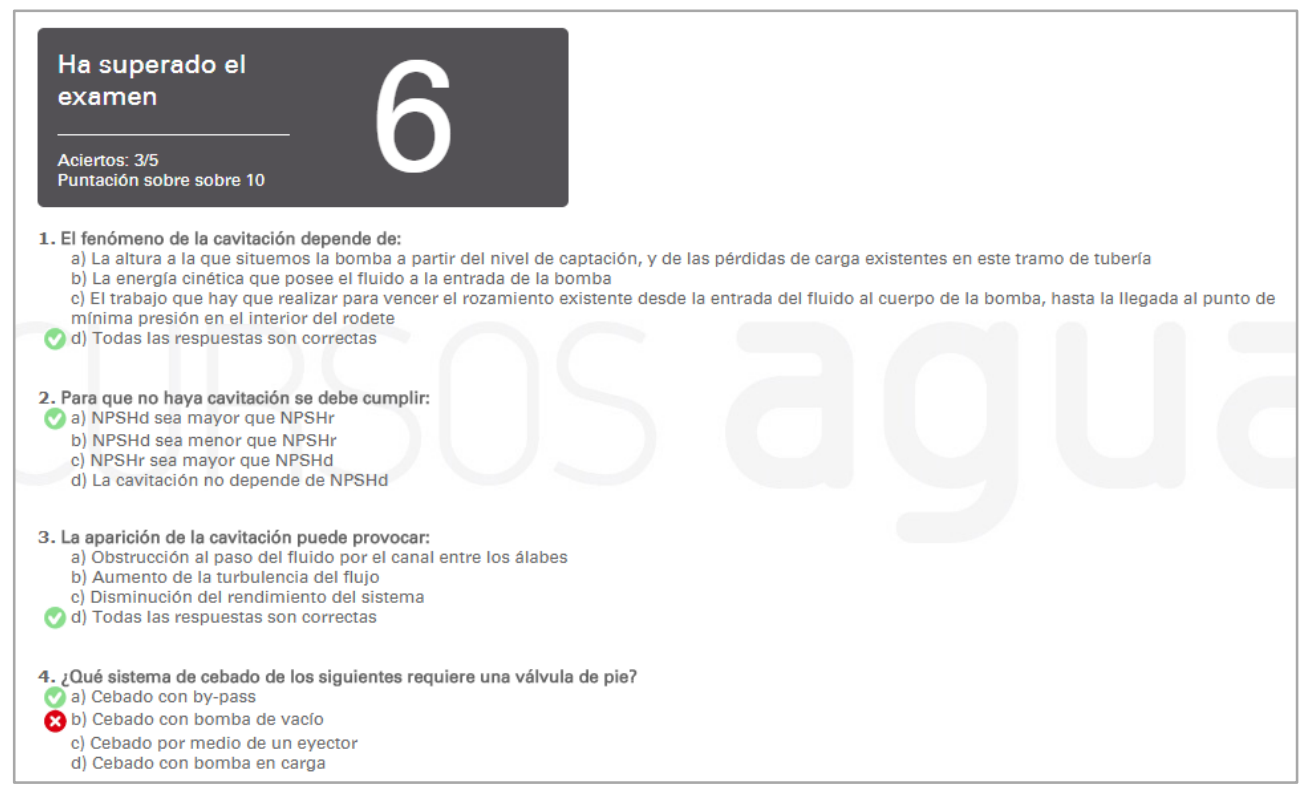

Fig. 3 Consulta de un test de unidad una vez finalizado

El alumno no está obligado a responder el test de la unidad una vez la haya finalizado, puede continuar con el desarrollo del curso y realizarlo posteriormente. De esta manera el alumno es el que decide cuando está preparado para presentarse a estos test, ya que en ocasiones el estudio del resto de unidades facilita la asimilación y comprensión de algunos conceptos.

(cc) EY-NC-ND 2018, Universitat Politècnica de València

Congreso IN-RED (2018) 
El peso del test de las unidades varía en función de si el curso requiere el envío de ejercicios o no. En el caso de no existir envío de ejercicios, los test de las unidades representan el 30\% de la evaluación final. Mientras que en los cursos con envíos de caso prácticos representan el $10 \%$ de la nota total, ya que se le da un mayor peso a este tipo de evaluación.

\subsection{Test final del curso}

Una vez desarrollados los contenidos del curso, con sus correspondientes puntos de control, así como los test de cada una de las unidades, se habilita automáticamente en la plataforma el test final del curso. Éste es similar a los test de las unidades, con preguntas multirespuesta de tipo teórico en las que el alumno debe elegir la respuesta correcta entre varias opciones, la diferencia radica en que en esta ocasión el test engloba todo el contenido desarrollado.

El número de preguntas del test final varía entre 10 y 20 preguntas. Al igual que en los test de las unidades, cada pregunta errada no descuenta, únicamente se puntúa con cero. En este caso, tanto en los cursos con envío de ejercicios como en los que no se requiere el envío de casos resueltos, el test final representa el $40 \%$ de la nota del curso. Las preguntas que aparecerán en este test final son preguntas esenciales para la superación del curso. Por esta razón, el test final tiene un peso importante dentro de la nota final. No superarlo dificulta notablemente aprobar el curso.

Al igual que en los test de las unidades, una vez culminado el test final, el alumno puede consultar la nota y los fallos y aciertos realizados, el aspecto es similar al del test que aparece en la Fig. 3.

\subsection{Entrega de ejercicios}

A lo largo de las unidades el alumno puede encontrarse con ejercicios o casos prácticos guiados. Normalmente este tipo de actividades se centran en la última unidad del curso, una vez desarrollados todos los conceptos teóricos, y realizados los casos prácticos guiados que van surgiendo en las diferentes unidades. No obstante, existen cursos muy prácticos relacionados con programas informáticos sobre hidráulica en los que el alumno deberá resolver ejercicios a lo largo de todo el curso, sea cual sea la unidad. No todos los cursos tienen ejercicios, siendo característicos de los cursos más prácticos.

$\mathrm{Al}$ alumno se le plantea el enunciado y las actividades prácticas a resolver en el ejercicio. Estos ejercicios deben ser resueltos y enviados a los tutores del curso junto con un informe que describa el proceso seguido para su resolución, y cualquier comentario al respecto que desee realizar el estudiante. En cualquier momento se puede solicitar una aclaración o ayuda al tutor del curso, tanto mientras desarrolla los contenidos como mientras resuelve el ejercicio, pero debe existir un envío final de cada ejercicio junto con el informe correspondiente que será el que sea evaluado, corregido y comentado con el alumno (Seoane y García, 2018, Bailey y Card, 2009).

Si el ejercicio no ha sido resuelto correctamente, se solicitará al alumno que vuelva a enviarlo dándole una serie de instrucciones para poder resolverlo de manera adecuada. El 
ejercicio no se puntuará hasta que el alumno lo haya enviado correctamente, el mero envío del ejercicio no da derecho a su evaluación. De esta manera, el alumno trabaja con el caso práctico planteado una y otra vez hasta dar con la solución, siempre bajo la supervisión y seguimiento del tutor. La valoración de los ejercicios depende del tutor, basándose en criterios objetivos y en la cantidad de cuestiones y envíos que haya realizado el alumno hasta la correcta solución.

Los ejercicios representan el $40 \%$ de la nota final del curso, siendo un peso muy importante en aquellos cursos prácticos donde se busca que el alumno trabaje de manera individual con la resolución de los ejercicios propuestos.

El alumno podrá responder el test final del curso antes del envío de los ejercicios solicitados, no obstante, el curso no se dará por terminado hasta la entrega de los ejercicios. Por lo tanto, aunque el alumno esté aprobado con una nota superior a 5 sin enviar los ejercicios, no se dará por superado el mismo hasta la correcta resolución de los ejercicios.

\section{Apartado de evaluaciones}

En todo momento el alumno tiene acceso al progreso de sus notas desde la propia plataforma donde se desarrolla el curso. Este apartado de notas y evaluaciones ha sido creado y desarrollado específicamente para implantar la metodología de evaluación descrita en este trabajo, de manera que el alumno se encuentra con una interfaz accesible y amigable que le permite consultar el porcentaje de evaluaciones realizadas, el peso de cada una de ellas respecto a la nota final y la nota obtenida en cada evaluación ya realizada.

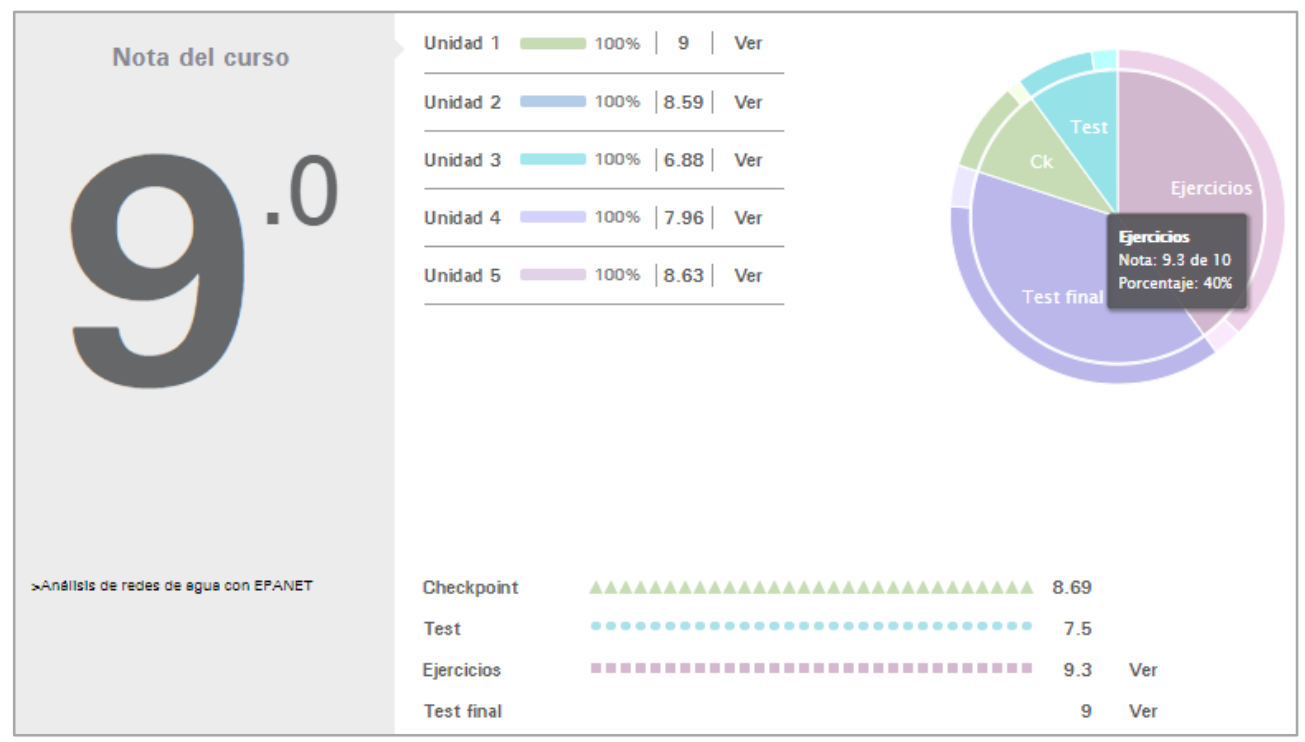

Fig. 4 Pantalla de notas y evaluaciones del curso en la Plataforma Cursosagua

Al comienzo del curso se parte de una nota de cero y se van sumando puntos progresivamente conforme se realizan las diferentes evaluaciones antes explicadas. Tal y como muestra la Fig. 4, en la parte izquierda de la pantalla evaluaciones, en números

(cc) EY-NC-ND 2018, Universitat Politècnica de València

Congreso IN-RED (2018) 
grandes, aparece la nota que tiene el alumno en cada momento. Una vez finalizado el curso y todas sus evaluaciones, esta nota representará la nota final de la formación sobre 10.

Desde este apartado de evaluaciones se accede a los test de cada unidad y al test final del curso, habilitándose progresivamente según se van finalizando las unidades. De esta manera el alumno no puede realizar el test de una unidad que previamente no haya finalizado.

Además de la nota global del curso, en esta ventana el alumno encuentra la nota desglosada por unidades, como se puede visualizar en la Fig. 5, donde se puede consultar la nota obtenida en el test y en los puntos de control que tiene la unidad, permitiendo el acceso a su consulta desde el mismo apartado, para repasar en qué cuestiones ha fallado y acertado.

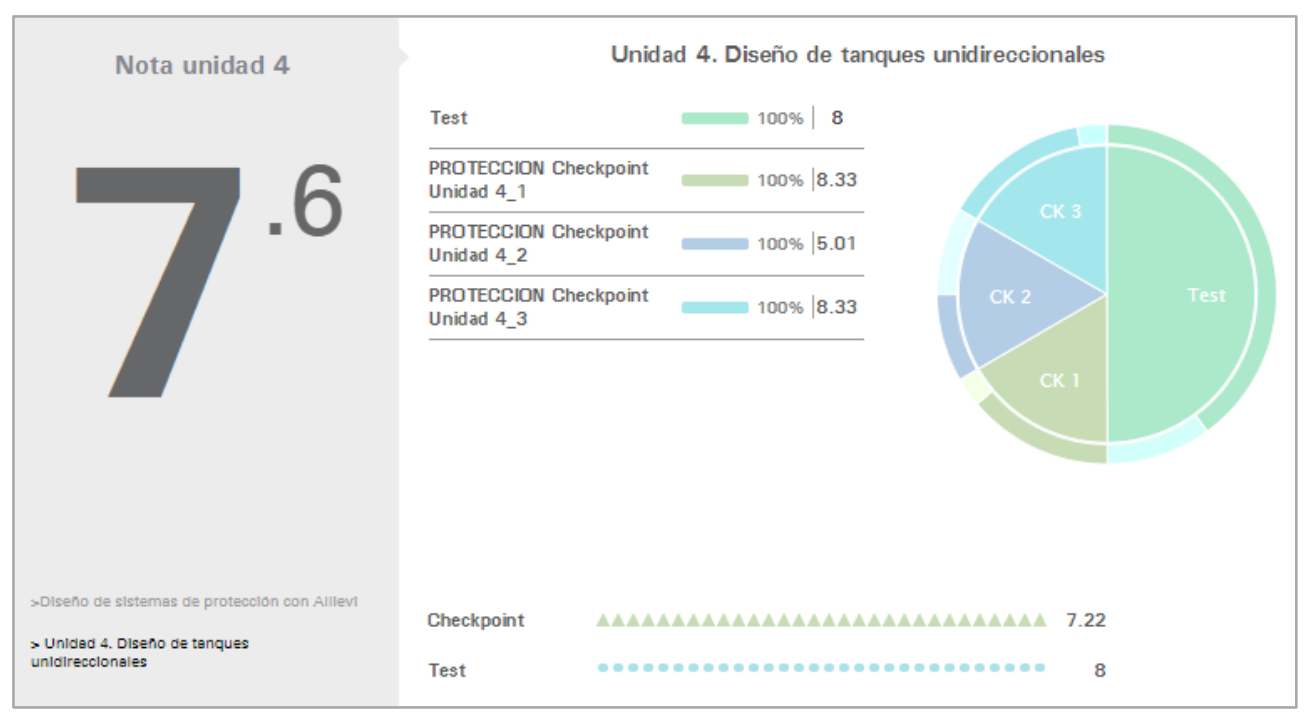

Fig. 5 Pantalla de notas y evaluaciones de una unidad en la Plataforma Cursosagua

Un gráfico circular a la derecha superior de la pantalla permite tener una primera visualización de las notas obtenidas en cada evaluación y el peso que representa cada una respecto a la nota final. Al pasar el cursor por cada una de las partes aparecerá un cuadro de texto indicando la nota y el porcentaje que representa sobre el total.

Finalmente, en la parte inferior de la pantalla, se visualiza un resumen de las notas obtenidas en cada una de las partes, pudiendo acceder desde aquí a la nota obtenida en cada uno de los ejercicios y al test final del curso.

\section{Resultados}

Los resultados de la metodología utilizada para evaluar los conocimientos adquiridos por los alumnos que realizan los cursos de formación a distancia impartidos por el ITA y que conducen a la obtención de títulos propios, son magníficos, con una tasa de aprobados superior al $90 \%$.

Los resultados de las encuestas realizadas desde el Centro de Formación Permanente (CFP) de la UPV sobre estos cursos, son muy positivos tal y como se observa en la Fig. 6, 
especialmente en lo referente a la evaluación de los contenidos: preguntas F, H e I, detalladas a continuación:

F. El módulo/asignatura ha incluido la realización de ejercicios, casos prácticos o simulaciones que han reforzado la adquisición de conocimientos

H. Las actividades y tareas (la carga de trabajo del módulo/asignatura en general) a lo largo del curso han estado adecuadamente distribuidas

I. Considero que el sistema de evaluación empleado ha sido el adecuado

\section{COMPARATIVA DE LAS PREGUNTAS}

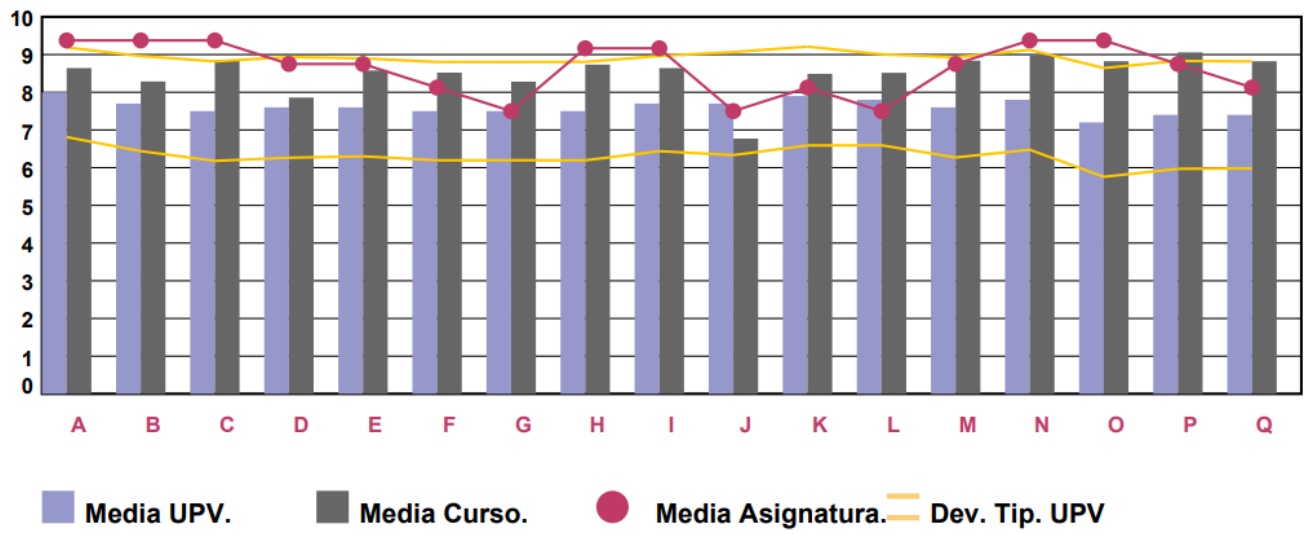

Fig. 6 Resultado de las encuestas de la asignatura Análisis de redes de abastecimiento de agua

Asimismo, en las encuestas de satisfacción realizadas por los alumnos a través del CFP, se refleja la satisfacción del alumnado con la metodología utilizada y la organización general del curso, con unos resultados por encima de la media de los cursos impartidos por la UPV, donde la satisfacción media de los alumnos a lo largo de las diferentes ediciones celebradas es superior a 9 sobre 10 tal y como se puede observar en la Fig.7. 
COMPARATIVA DE LAS PREGUNTAS

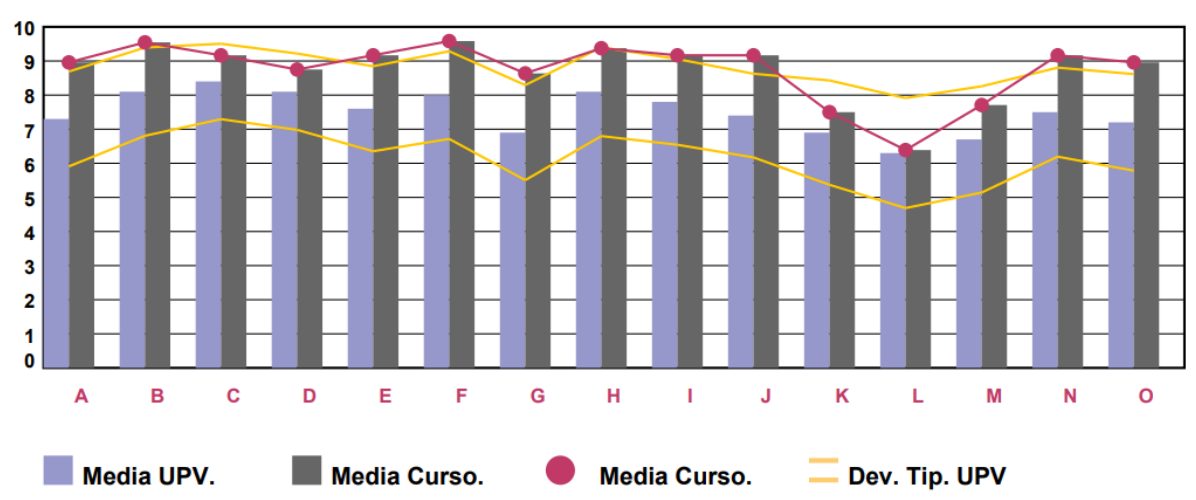

Enunciados de las preguntas:

\begin{tabular}{lr}
\hline A. La organización general del curso es buena. & 9 \\
\hline B. Puedo contactar con la dirección/coordinación del curso siempre que lo solicito. & $\mathbf{9 , 5}$ \\
\hline C. El trato recibido por parte del equipo de dirección/coordinación del curso es correcto & $\mathbf{9 , 2}$ \\
\hline D. En términos generales, la relación profesor-alumno es buena. & $\mathbf{8 , 8}$ \\
\hline E. El material de promoción consultado tenía la información suficiente. & $\mathbf{9 , 2}$ \\
\hline F. El horario actual del curso se adapta a mi disponibilidad. & $\mathbf{9 , 6}$ \\
\hline G. Hay suficiente coordinación entre las diferentes asignaturas. & $\mathbf{8 , 6}$ \\
\hline H. Considero que la realización del curso puede ser útil en mi campo profesional. & $\mathbf{9 , 4}$ \\
\hline I. En términos generales, la documentación entregada en el curso es útil para mi. & 9,2 \\
\hline J. Los métodos de enseñanza utilizados me parecen adecuados el tipo de curso. & $\mathbf{9 , 2}$ \\
\hline K. Las actividades complementarias (visitas a empresas, etc.), en caso de haberse & $\mathbf{7 , 5}$ \\
\hline organizado, me parecen interesantes. & $\mathbf{6 , 4}$ \\
\hline L. La realización del curso me ayuda a crear relaciones profesionales útiles. & $\mathbf{7 , 7}$ \\
\hline M. La relación entre la calidad del curso y su precio de inscripción me parece adecuada. & $\mathbf{9 , 2}$ \\
\hline N. Opino que la calidad del curso es buena. & $\mathbf{9}$ \\
\hline O. Mis expectativas ante el curso quedan satisfechas. &
\end{tabular}

Fig. 7 Resultado de las encuestas del título propio de posgrado:Máster en gestión eficiente del agua urbana

El sistema de evaluación junto con los excelentes resultados obtenidos por los alumnos ha suscitado un creciente interés por las empresas para formar a sus empleados, ya que permite una flexibilidad y seguimiento de los contenidos compatible con las tareas laborales. La Fig. 8 muestra el aumento de alumnos matriculados pertenecientes a empresas. No hay que olvidar que el acceso a la plataforma es de 24 h los 365 días al año, permitiendo a todos los alumnos acceder cuando mejor les convenga, sin restricciones horarias. 


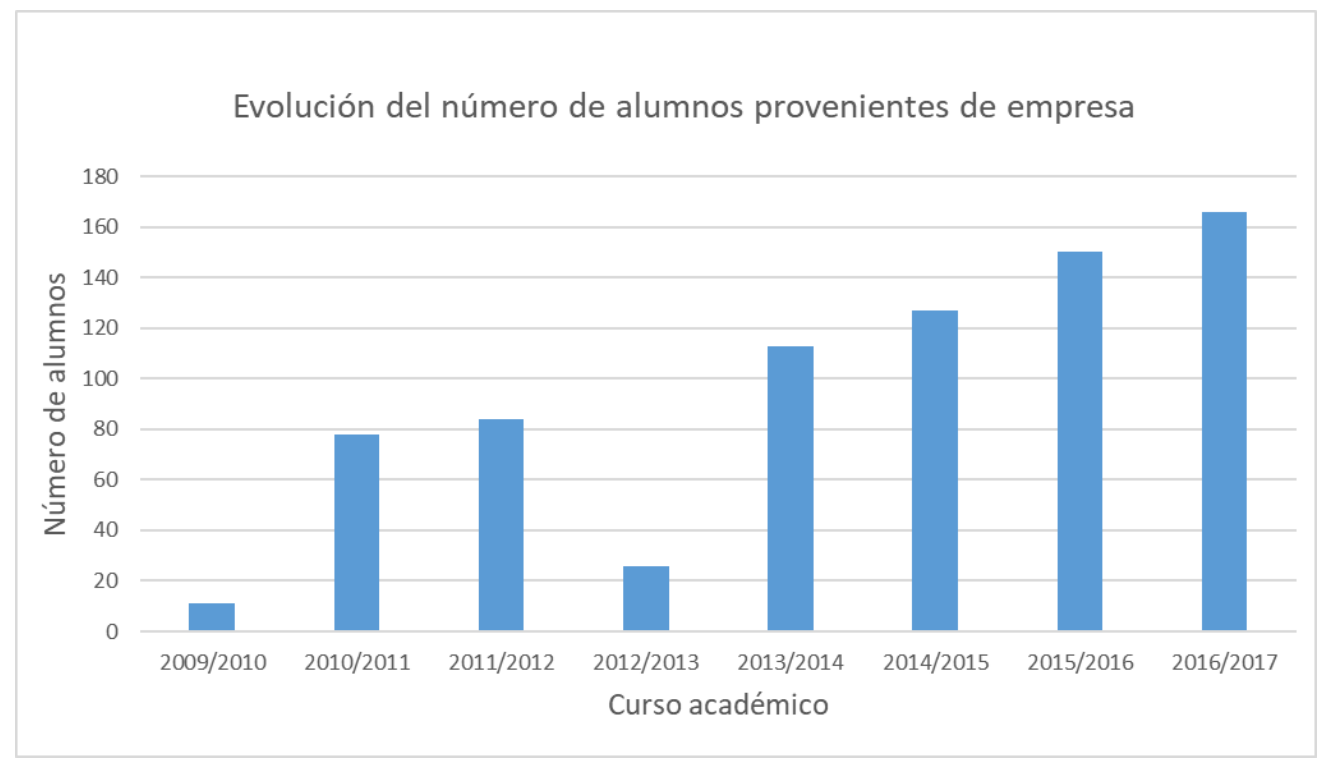

Fig. 8 Evolución del número de alumnos provenientes de empresas

La calidad en la formación a distancia del ITA se refleja no sólo en la excelente puntuación obtenida en las encuestas, sino también a través del creciente número de alumnos matriculados y del número de módulos realizados, ya que muchos de éstos optan por ampliar su formación con el ITA. La Fig.9 muestra el aumento de módulos cursados. Se puede observar como en el último año académico se ha casi duplicado este valor. Esto es debido a la gran aceptación que han tenido los cuatro títulos de posgrado que se imparten desde el curso académico 2016/2017.

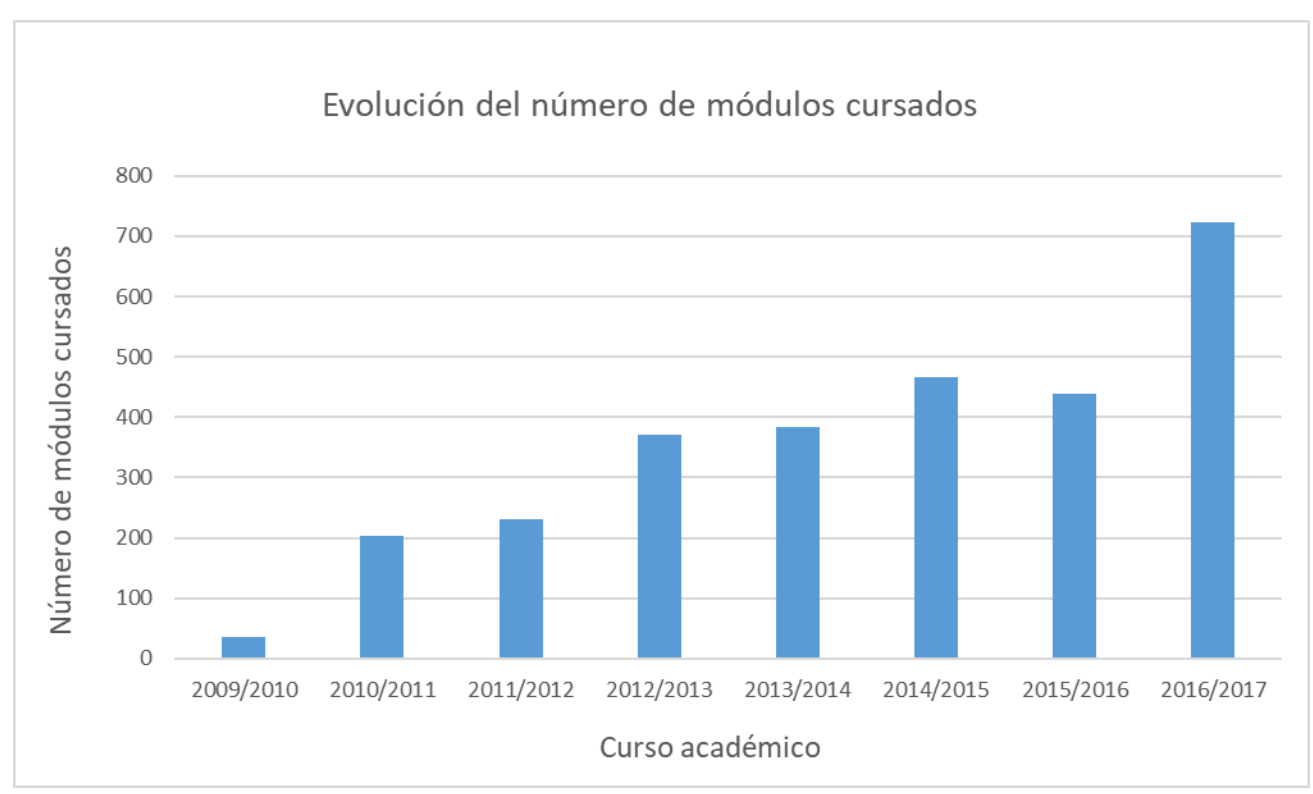

Fig. 9 Evolución del número de módulos cursados

(c)) EY-NC-ND 2018, Universitat Politècnica de València

Congreso IN-RED (2018) 


\section{Conclusiones}

El sistema de evaluación presentado está diseñado para asegurar que los alumnos consiguen superar el curso cumpliendo con los requisitos mínimos exigidos, siempre más difíciles de evaluar en formación a distancia.

Esta tarea de asegurar que el alumno dispone de los conocimientos estudiados una vez finaliza la formación, es vital en formaciones técnicas, más aún en el campo de la hidráulica, la modelación de redes y la gestión eficiente de agua, pues suponen conocimientos muy concretos de aplicabilidad directa a casos reales, que usualmente suelen servir de acceso a puestos de trabajo de carácter técnico y especializado.

Este objetivo se cumple con este sistema de evaluación, ratificado por el creciente número de empresas interesadas en formar a sus empleados aplicando este sistema, y por las encuestas de satisfacción realizadas por los alumnos una vez finalizado el curso.

\section{Referencias}

BAILEY, C. J., CARD, K. A. (2009). "Effective pedagogical practices for online teaching: Perception of experienced instructors”. The Internet and Higher Education, 12(3-4), 152-155.

BHUASIRI W., XAYMOUNGKHOUN O., ZO H., JAE JEUNG RHO J., CIGANEK A. (2011). "Critical success factors for e-learning in developing countries: A comparative analysis between ICT experts and faculty” Computers \& Education 58(2):843-855 (February 2012) DOI10.1016/j.compedu.2011.10.010

FRYDENBERG, J. (2002). “Quality standards in eLearning: A matrix of analysis”. The International Review of Research in Open and Distributed Learning, 3(2).

GRUPO DE INVESTIGACIÓN EN INTERACCIÓN Y ELEARNING (GRIAL). UNIVERSIDAD DE SALAMANCA. "Manual de Tutoría Online”.

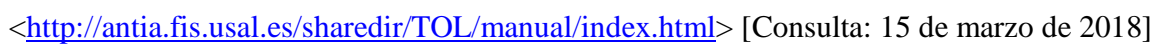
[Antonio M. Seoane Pardo y Francisco J. García Peñalvo]

MENDES, A.C., CURTO, S., COHEUR, L. (2011). "Bootstrapping multiple-choice tests with thementor”. Computational Linguistics and Intelligent Text Processing, 451-462. Heidelberg: Springer.

SINGH, U.G., (RUTH) DE VILLIERS, M.R., (2017). “An evaluation framework and instrument for Evaluating e-Assessment Tools”. International Review of Research in Open and Distance Learning, 18(6), pp.164-185.

SUN P., RAY J. TSAI R., FINGER G., CHEN Y., DOWMING Y. (2006). "What drives a successful e-Learning? An empirical investigation of the critical factors influencing learner satisfaction" en Computers \& Education 50(4):1183-1202. DOI10.1016/j.compedu.2006.11.007

VENTOURAS, E., TRIANTIS, D., TSIAKAS, P., STERGIOPOULOS, C. (2010). "Comparison of examination methods based on multiple choice questions and constructed-response questions using personal computers”. Computers \& Education, 54(2), 455-461. 
VICERRECTORADO DE CALIDAD. UNIVERSIDAD DE EXTREMADURA. "Consejos para la creación y gestión de MOOC - Miriada X".

$<$ https://www.unex.es/organizacion/gobierno/vicerrectorados/vicecal/archivos/ficheros/moocs /Consejos_rapidos_para_formato_MOOC.pdf/view> [Consulta: 20 de mayo de 2018] [Ángel González de la Fuente y David Carabantes Alarcón]

(cc) EY-NC-ND 2018, Universitat Politècnica de València 